\title{
Correction: Assuring safe patient care in a level III NICU in anticipation of hospital closure
}

\author{
Rachel Fleishman (1) - Endla Anday • Vineet Bhandari
}

Published online: 23 April 2020

(c) Springer Nature America, Inc. 2020

Correction to: Journal of Perinatology

https://doi.org/10.1038/s41372-020-0648-7

published online 17 March 2020
At the request of the VON, based on their data sharing policy, we have removed the VON reference in the abstract, the VON comparison data from Table 2, adjusted the "Results" section that compares our data to VON, and added the verbiage requested by them in "Acknowledgements". 